\title{
INVESTIGATION ON EVOLUTIONARY EDTAS CHAOS CONTROL
}

\author{
Roman Senkerik \\ Ivan Zelinka \\ Eduard Navratil \\ Department of Applied Informatics \\ Faculty of Applied Informatics, Tomas Bata University in Zlin \\ Nad Stranemi 4511, Zlin 762 72, Czech Republic \\ E-mail: \{senkerik, zelinka, enavratil\}@fai.utb.cz
}

\section{KEYWORDS}

Chaos, control, optimization, evolutionary algorithms, parameter estimation

\begin{abstract}
This work deals with an investigation on optimization of the feedback control of chaos based on using of the evolutionary algorithms. The main aim of this work is to show that evolutionary algorithms are capable of optimization of chaos control. As a model of deterministic chaotic system the Henon map was used. The optimizations were realized in several ways, each one for another set of parameters of evolution algorithms or another cost functions. The evolutionary algorithm SOMA (Self-Organizing Migrating Algorithm) was used in four versions. For each version simulations were repeated several times to show and check robustness of used method.
\end{abstract}

\section{INTRODUCTION}

Since the early 1990's many methods for control of chaos using the feedback control scheme have been developed and based on the OGY control method (Ott, et al. 1990). They are for example special modified versions of OGY, including pole placement method (Boccaletti, et al. 2000), targeting algorithms or delayed feedback control. (Just 1999) An advantage of the OGY method and all of its variations is that it does not require a priori knowledge of the equations of the system. But on the other hand there is generally one big disadvantage and it is the long initial chaotic transient before trajectories are stabilized. Consequently many targeting algorithms were introduced to shorten the time of stabilization. Unlike OGY the Pyragas method based on the delayed feedback control can be simply considered as targeting and stabilizing algorithm together in one package (Kwon 1999). See Fig. 1. as the example of the difference between OGY and delay feedback control. Another big advantage of Pyragas method is an amount of accessible control parameters, which are set up by using a priori knowledge or mathematical analysis. This is very advantageous for successful use of optimization of parameters set up, leading to improvement of system behavior and better and faster stabilization to the desired periodic orbits.
Some research in this field has been recently done using the evolutionary algorithms for optimization of local control of chaos based on a Lyapunov approach (Richter and Reinschke, 2000).
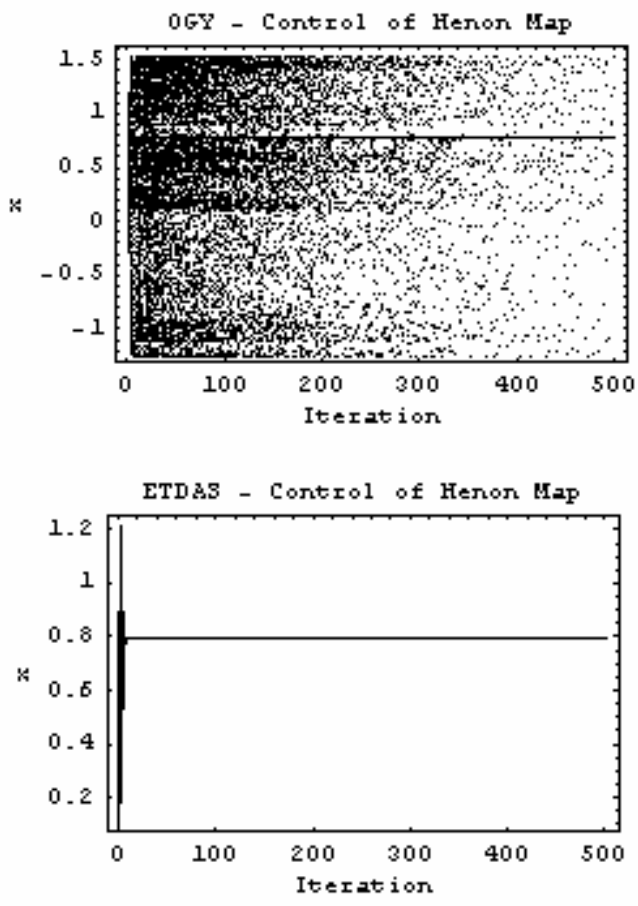

Fig. 1. Comparison of control to fixed point for OGY and ETDAS method.

This contribution deals with an investigation on the optimization of the control of chaos by the evolutionary algorithms (EA). The control law is based on Pyragas method: Extended delay feedback control, also called Extended time delay auto synchronization - ETDAS (Pyragas 1995), which was developed from the original Pyragas method - time delay auto synchronization TDAS (Pyragas 1992).

This contribution is continuation of previous experiments with application of EA to chaos control (Zelinka 2005a, Zelinka 2005b) The main aim of this work is to show that EA are capable to search for optimal set up of control parameters without internal system knowledge and operate with deterministic chaos as with black box. 


\section{PROBLEM DESIGN}

\section{Problem selection and case studies}

The chosen example of chaotic system was two dimensional Henon map in form (1).

$$
\begin{aligned}
& x_{n+1}=a-x_{n}^{2}+b y_{n} \\
& y_{n+1}=x_{n}
\end{aligned}
$$

Due to the reasons described above ETDAS control algorithm was used here. This work consists of several case studies. Each one is focused on estimation of three accessible control parameters for EDTAS method to stabilize desired unstable periodic orbit and comparison of results of used versions of EA. Desired unstable periodic orbit are following: $1 p, 2 p, 4 p$ orbit and finally the study of possibility of control to higher periodic orbits (6p and 8p) All simulations were based on the same model and several times repeated for each EA, (100 or 50 times, this depends on the used method and time demands), with new initial conditions for each simulation to show and check robustness of used methods. All the important results of the best simulations for both algorithms are discussed continuously to show the behaviour of the system and control method with optimized parameters set up and its capability of the stabilization of periodic orbits for initial conditions uniformly distributed in the region of $0<x_{\text {initial }}=y_{\text {initial }}<1$. The control method - ETDAS has form (2).

$$
\begin{gathered}
\frac{d x}{d t}=P(x)+F(t) \\
F(t)=K[(1-R) S(t-\tau)-x(t)] \\
S(t)=x(t)+R S(t-\tau)
\end{gathered}
$$

Where $K$ and $R$ are experimentally adjustable constants, $F$ is the perturbation and $S$ is given by a delay equation utilizing previous states of the system. After modification into discrete form suitable for two dimensional Henon map based on (Mensour and Longtin, 1998), control algorithm ETDAS is obtained in form (3)

$$
\begin{gathered}
x_{n+1}=a-x_{n}^{2}+b y_{n}+F_{n} \\
F_{n}=K\left[(1-R) S_{n-m}-x_{n}\right] \\
S_{n}=x_{n}+R S_{n-m}
\end{gathered}
$$

Where $m$ is the period of $m$-periodic orbit to be stabilized. The perturbation $F_{n}$ in equations (3) may have arbitrarily large absolute value, which is not physically sensible. Therefore, $F_{n}$ should have a value between $-F_{\max }$ and $F_{\max }$.

\section{The cost function}

The cost function $(\mathrm{CF})$ has been calculated from the distance between desired state and actual system output. The minimal value of this cost function revealing the best solution is 0 . The aim of all the simulations was to find the best solution that returns the cost function value as close as possible to 0 . But it was possible to use this $\mathrm{CF}$ (4) only for the stabilization of p-1 orbit, thus to minimize the area created by the difference between the required state (stabilized fixed point) and the real system output on the simulation interval $-\tau$. Another cost function was used for stabilizing of higher periodic orbit. It was synthesized from cost function (4) and other terms had to be added. This CF was in general based on searching for desired periodic orbit and calculation of the difference between desired and real periodic orbit.

$$
f_{\cos t}=\sum_{t=0}^{\tau}\left|T S_{t}-A S_{t}\right|
$$

Where: TS - target state

AS - actual state

\section{Optimization algorithms}

For the experiments described here stochastic optimization algorithm SOMA (Zelinka, 2004), has been used. SOMA is modeled on the social behaviour of co-operating individuals. It was chosen because it has been proven that the algorithm has the ability to converge towards the global optimum.

The question why the evolutionary algorithms were used for optimizing of control of chaos is answered in Fig. 2 and Fig. 3. The first one shows the illustrative example of dependence of the CF (4) on the value of adjustable parameter $K$. And the second one shows the dependence of CF value on parameters $K$ and $F_{\max }$ for p- 1 orbit. The CF surface is highly nonlinear and erratic thus it is not possible to use common deterministically based search methods. This kind of surfaces can be successfully searched by evolutionary algorithms.

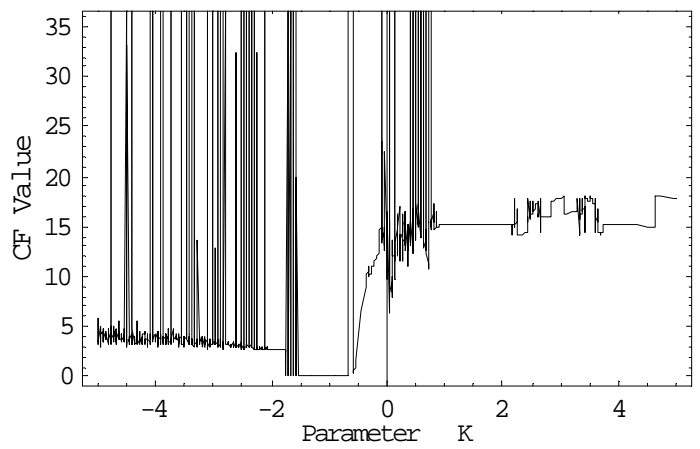

Fig. 2. Dependence of CF value on parameter $K$ for p-1 orbit, $x_{\text {initial }}=0.7, F_{\max }=0.4492, R=0.3269$ 


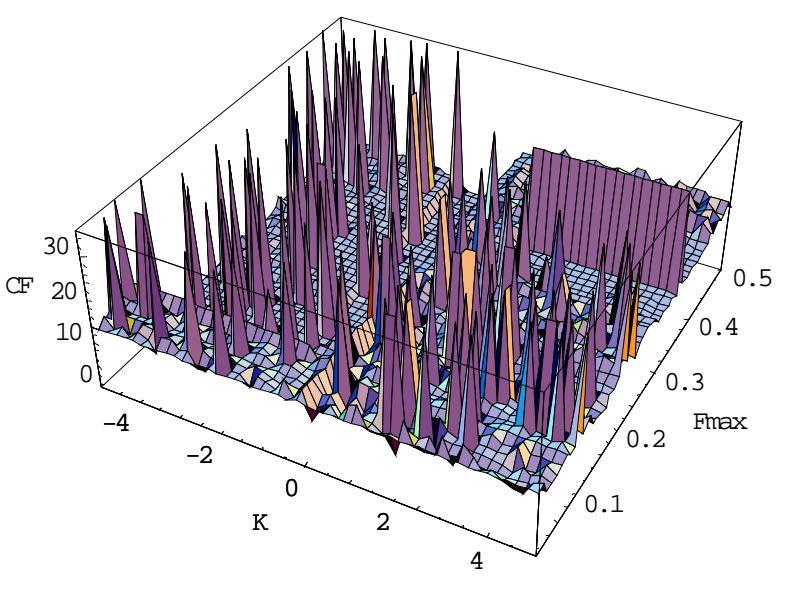

Fig. 3. Dependence of CF value on parameters $K$ and $F_{\max }$ for p-1 orbit, $x_{\text {initial }}=0.7, R=0.3269$ (With higher resolution the complexity of this figure rapidly increases.)

\section{Parameter settings for optimization algorithms}

Four versions of SOMA were used for all the simulations. See Tab. 1. for relation between each version and index mark in figures $4-13$. Parameters for optimizing algorithm were set up in this way in order to reach the same value of maximal $\mathrm{CF}$ evaluations, see Tab. 2. To avoid very long time demanding simulations a decision rule was added into the $\mathrm{CF}$. This rule stopped EA immediately, when the first individual with good parameter structure was reached thus the value of $\mathrm{CF}$ was lower then acceptable one (typically $\left.\mathrm{CF}_{\mathrm{acc}}=0.001\right)$. The ranges of all estimated parameters were these:

$$
-5 \leq K \leq 5,0 \leq F_{\max } \leq 0.5 \text { and } 0 \leq R \leq 1
$$

Tab.1 Used versions of SOMA

\begin{tabular}{|c|c|}
\hline Index & Algorithm / Version \\
\hline 1 & SOMA AllToOne \\
\hline 2 & SOMA AllToRandom \\
\hline 3 & SOMA AllToAll \\
\hline 4 & SOMA AllToAllAdaptive \\
\hline
\end{tabular}

Tab.2 Parameter settings for SOMA

\begin{tabular}{|l|c|c|}
\hline \multicolumn{1}{|c|}{ Parameter } & ATO / ATR & ATA / ATAA \\
\hline PathLength & 3 & 3 \\
\hline Step & 0.33 & 0.33 \\
\hline PRT & 0.1 & 0.1 \\
\hline PopSize & 25 & 10 \\
\hline Migrations & 25 & 7 \\
\hline $\begin{array}{l}\text { Max. CF } \\
\text { Evaluations } \\
\text { (CFE) }\end{array}$ & 5400 & 5670 \\
\hline
\end{tabular}

\section{EXPERIMENTAL RESULTS}

Each version of SOMA has been applied many times with new initial conditions for each simulation in order to find the actual optimum. The primary aim of this contribution is not to show which version is better or worse but to show that the EA can be really used for deterministic chaos control when the cost function is properly defined. Outputs of simulations are depicted in Figures 4 - 13. The best individual solutions together with comparison of SOMA versions are shown for each case study - desired periodic orbit. All results are shown only for variable $x$ of two dimensional Henon map because of its form (1), where the variable $y$ has the same values as variable $x$ but it is only phase shifted

\section{Estimation of control parameters for p-1 orbit}

In the first case the point was to estimate the optimum value of feedback adjustable constants to stabilize $1 \mathrm{p}$ orbit (a fixed point). Unperturbed Henon map $\left(F_{\mathrm{n}}=0\right)$ with parameters $a=1.2$ and $b=0.3$ has the fixed point $x_{F}=0.8$.

The best results of each version of SOMA are shown in Tab. 3. Comparison of SOMA versions from the point of view of $\mathrm{CF}$ evaluations (CFE), and estimated parameters $K, F_{\max }, R$ is in Fig. 4. These diagrams show the variance from min. to max. of observed parameter and the small rectangular mark represents average value. Each SOMA version gave the same result of $\mathrm{CF}$ value for the best solution. But as can be seen from histograms (Fig. 5), SOMA ATR has better distribution of CF values. See Fig. 6. for the best individual solution with the lowest $C F$ value and the same one iterated for initial conditions uniformly distributed is in Fig. 7.

Based on obtained results it may be stated that the control parameters estimated in the optimizations ensure very fast reaching of desired state for any initial conditions. And also all SOMA versions needed, on average, only $50 \mathrm{CF}$ evaluations to find a satisfactory solution. These facts show the possibility of using EA for real time chaos control to p-1 orbit (fixed point).

Tab.3 Best individual solutions for $\mathrm{p}-1$ orbit

\begin{tabular}{|c|c|c|c|c|}
\hline EA & $K$ & $F_{\max }$ & $R$ & CF Value \\
\hline 1 & -1.03809 & 0.449163 & 0.326949 & 0 \\
\hline 2 & -1.01208 & 0.142598 & 0.294697 & 0 \\
\hline 3 & -1.16149 & 0.316863 & 0.256766 & 0 \\
\hline 4 & -1.04145 & 0.155919 & 0.345818 & 0 \\
\hline
\end{tabular}



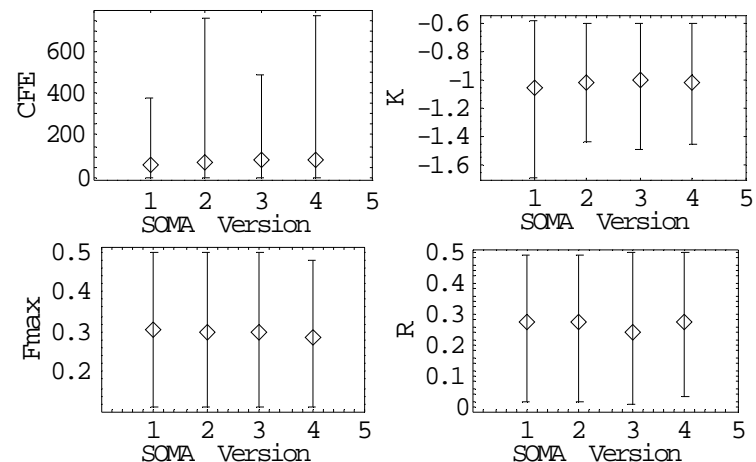

Fig. 4. Comparison of SOMA versions for CFE, and estimated parameters $K, F_{\max }, R$ for p-1 orbit
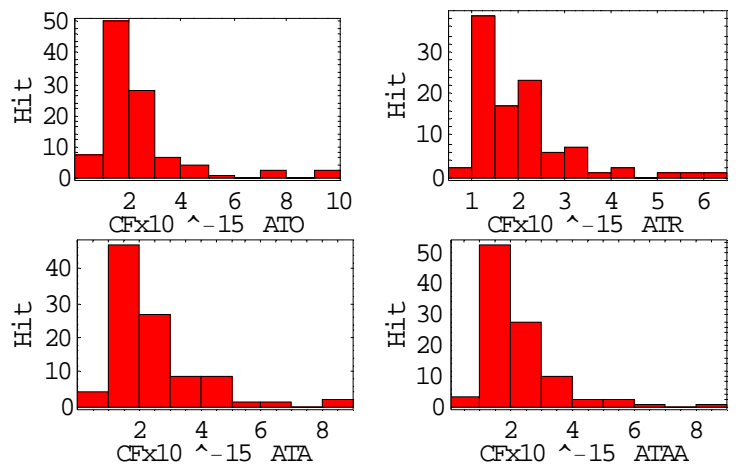

Fig. 5. Histograms for $\mathrm{p}-1$ orbit control optimization

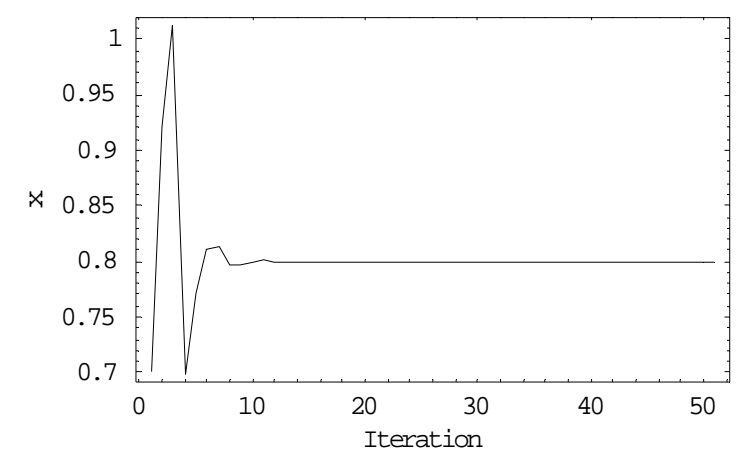

Fig. 6. Best individual solution: p-1 orbit, SOMA ATR

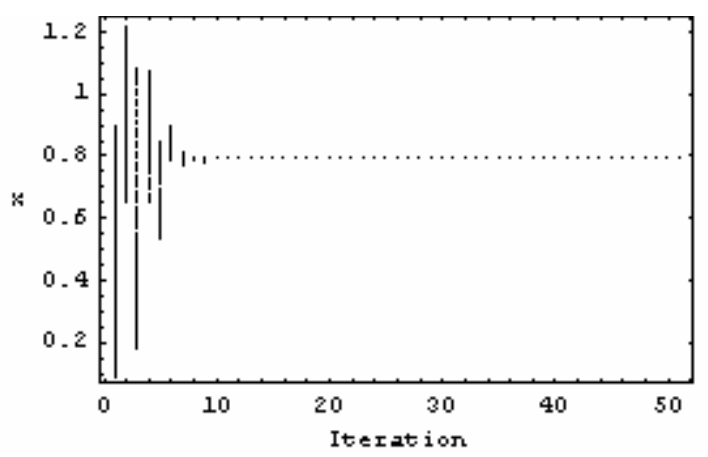

Fig. 7. Best solution for $\mathrm{p}-1$ orbit, SOMA ATR

\section{Estimation of control parameters for $\mathbf{p}-2$ orbit}

In this case the point was to reach the stabilization of $2 \mathrm{p}$-orbit. Unperturbed Henon map has this $\mathrm{p}-2$ orbit: $x_{1}=-0.562414, x_{2}=1.26241$.

The best results of each version of SOMA are shown in Tab. 4. Comparison of SOMA versions from the point of view of CF evaluations, and estimated parameters $K$, $F_{\text {max }}, R$ is in Fig. 8. See Fig. 9. and 10. for simulations of the best individual solution with the lowest $\mathrm{CF}$ value. Three SOMA versions gave almost same results of the best solution and as can be seen from Tab. 4, SOMA ATR has found the lowest value of CF. From the Fig. 9 follows that the stabilization was achieved relatively quickly and in the case of simulation for distributed initial conditions (Fig. 10) the desired periodic orbit was reached in first 500 iterations for more than $95 \%$ cases.

Tab.4 Best individual solutions for $\mathrm{p}-2$ orbit

\begin{tabular}{|c|c|c|c|c|}
\hline EA & $K$ & $F_{\max }$ & $R$ & CF Value \\
\hline 1 & 0.389335 & 0.09861 & 0.271823 & $2.17 \cdot 10^{-7}$ \\
\hline 2 & 0.472397 & 0.155925 & 0.461329 & $1.60 \cdot 10^{-14}$ \\
\hline 3 & 0.558013 & 0.15257 & 0.421521 & $1.57 \cdot 10^{-7}$ \\
\hline 4 & 0.54784 & 0.153437 & 0.43191 & $1.53 \cdot 10^{-8}$ \\
\hline
\end{tabular}
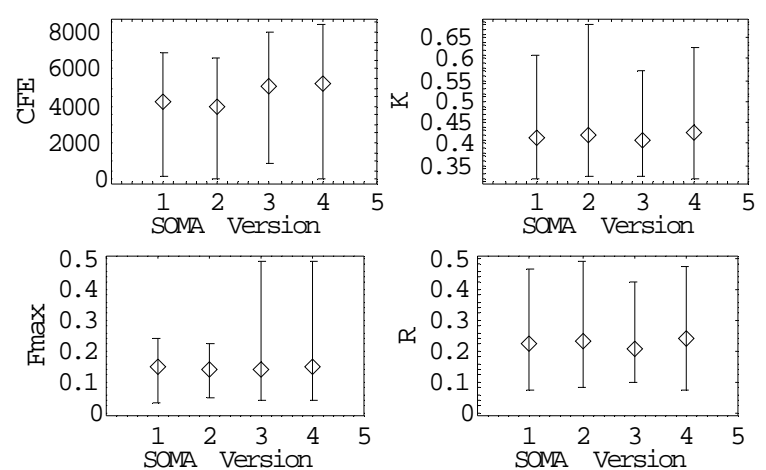

Fig. 8. Comparison of SOMA versions for CFE, and estimated parameters $K, F_{\max }, R$ for $\mathrm{p}-2$ orbit

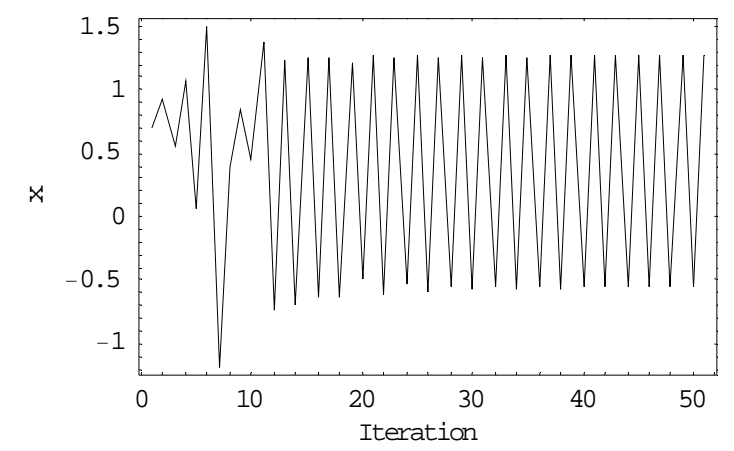

Fig. 9. Best individual solution: p-2 orbit, SOMA ATR 


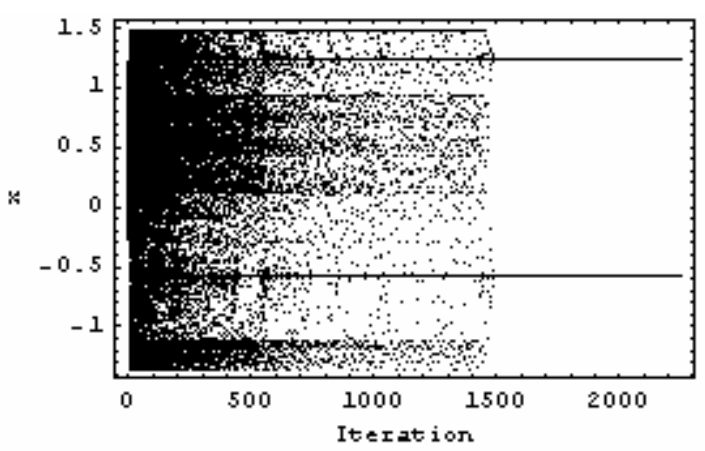

Fig. 10. Best solution for $\mathrm{p}-2$ orbit, SOMA ATR

\section{Estimation of control parameters for p-4 orbit}

The third case is focused on the stabilization of $\mathrm{p}-4$ orbit. Unperturbed Henon map has the following p-4 orbit:

$x_{1}=0.139, x_{2}=1.4495, x_{3}=-0.8595, x_{4}=0.8962$

The best results of each version of SOMA are shown in Tab. 5 .

From the comparison of SOMA versions (Fig. 11) it is obvious that for the stabilization of 4-p orbit more than $4000 \mathrm{CF}$ evaluations is needed as in previous case. This fact is related to highly increasing complexity of $\mathrm{CF}$ surface for higher period orbits. This kind of CF surface contains huge amount of local minimums and lots of them lead only to successful stabilization only for limited circle of initial conditions $x_{\text {initial }}$ This can be clearly seen in Fig. 12 which represents the best individual solution with the lowest $\mathrm{CF}$ value and in Fig. 13 where is the same solution iterated for initial conditions uniformly distributed in the region of $0<x_{\text {initial }}<1$. In the first case (Fig. 12) the same initial conditions were used for optimization process and for simulation of results, desired state was stabilized very quickly whereas in the second case (Fig. 13) for some initial conditions the stabilization is reached after more than 1500 iterations.

As a conclusion of this case study it is possible to say that all SOMA versions gave almost same results of the best solution but as can be seen from Tab. 5, SOMA ATAA has found the slightly lowest value of CF.

Tab.5 Best individual solutions for 4-p orbit

\begin{tabular}{|c|c|c|c|c|}
\hline EA & $K$ & $F_{\max }$ & $R$ & CF Value \\
\hline 1 & -0.383347 & 0.312323 & 0.436979 & $9.57 \cdot 10^{-8}$ \\
\hline 2 & -0.425825 & 0.283005 & 0.457182 & $5.39 \cdot 10^{-8}$ \\
\hline 3 & -0.369616 & 0.112991 & 0.410288 & $9.43 \cdot 10^{-9}$ \\
\hline 4 & -0.412404 & 0.341886 & 0.467282 & $9.90 \cdot 10^{-8}$ \\
\hline
\end{tabular}
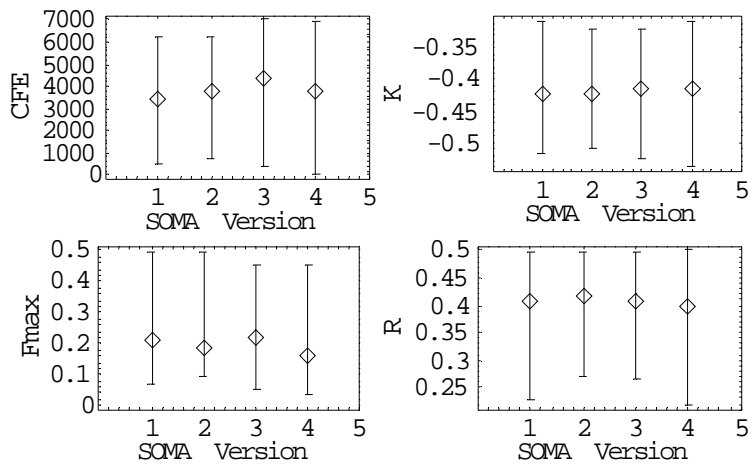

Fig. 11. Comparison of SOMA versions: CFE, and estimated parameters $K, F_{\max }, R$ for p-4 orbit

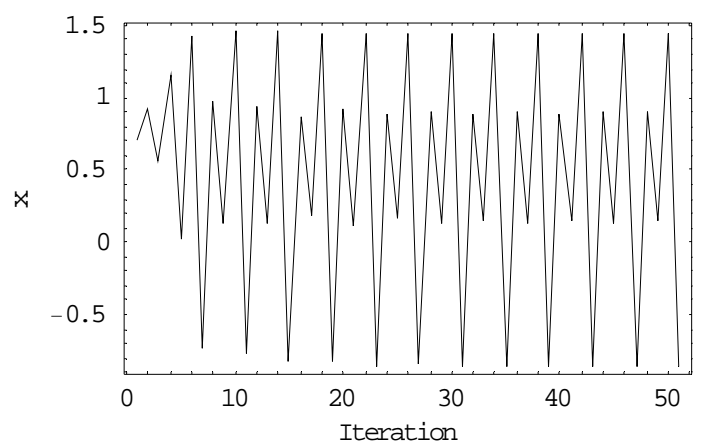

Fig. 12. Best individual solution: $\mathrm{p}-4$ orbit, SOMA ATA

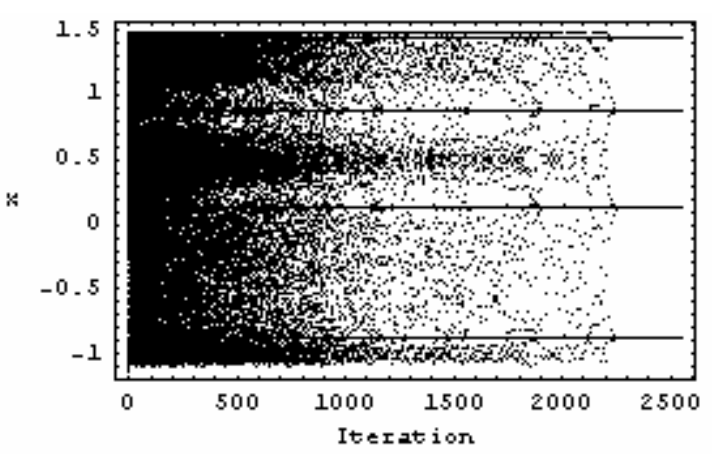

Fig. 13. Best solution for $\mathrm{p}-4$ orbit, SOMA ATA

\section{Estimation of control parameters for $\mathbf{6}$ and $\mathbf{8 p}$-orbit}

The last case is focused on the possibility of stabilization of higher periodic orbits $(6 \mathrm{p}$ and $8 \mathrm{p})$. As can be seen from Fig. 14 the CF surface is in this case highly nonlinear and highly erratic. Together with decreasing ability of ETDAS method to stabilize higher periodic orbits it was not possible to use the stopping rule in the $\mathrm{CF}$ as in the three previous cases. As the best $\mathrm{CF}$ value was not in any case lower then the acceptable one, all simulations were very time demanding. This problem may be solved soon by the changing of the $\mathrm{CF}$, parameters of EA or adding of extra adjustable constants into EDTAS control method. Unperturbed Henon map has many 6-p or 8-p orbits and it also depends on their degree of stability. 


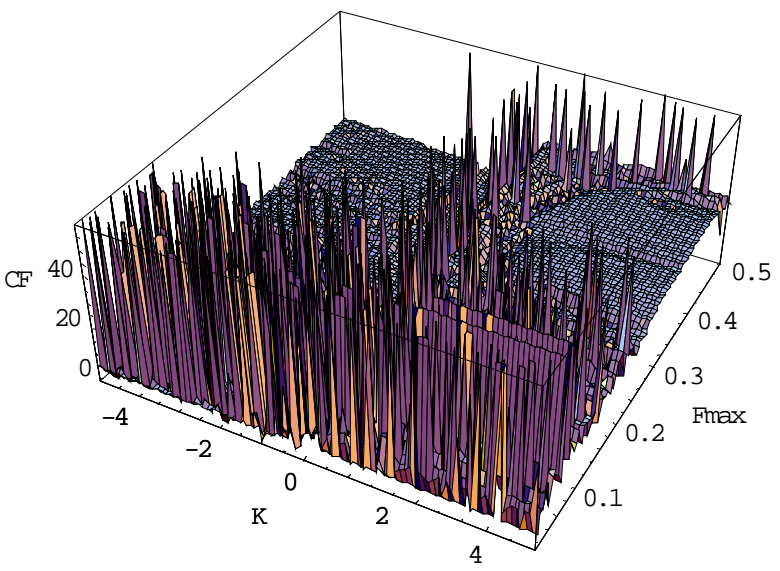

Fig. 14. Dependence of CF value on parameters $K$ and $F_{\text {max }}$ for p-6 orbit, $x_{\text {initial }}=0.7, R=0.3259$

\section{CONCLUSION}

Based on obtained results, it may be stated that all simulations give satisfactory results and thus evolutionary algorithms are capable of solving this class of difficult problems. Quality of results produced by given optimizations mostly depends on the definition of a given cost function. Its construction may comprise not only optimization of a basic criterion but also optimization of subcriteria capable of improving optimization quality. The main aim of this work was to show that EA are capable to solve chaos control problems. Each case study was focused on estimation of three accessible control parameters for EDTAS control method to stabilize desired unstable periodic orbits. And also these case studies were focused on comparison of results of used versions of EA. When comparing used four versions of SOMA, it is obvious that they all give very good results. Parameter settings for EA were based on heuristic approach and the CF was very simple, therefore there are possibilities for the future research in the field of evolutionary deterministic chaos control. According to all results showed here it is planned that the main activities would be focused on searching for better settings of EA, together with testing more complex cost functions.

\section{ACKNOWLEDGEMENT}

This work was supported by the grant NO. MSM 7088352101 of the Ministry of Education of the Czech republic and by grants of Grant Agency of Czech Republic GACR 102/06/1132 and GACR 102/05/0271.

\section{REFERENCES}

Boccaletti S., C. Grebogi, Y.C. Lai, H. Mancini and D. Maza 2000, The control of chaos: Theory and applications, Physics Reports, 329, 103-197.

Mensour B. and A. Longtin, 1998, Chaos control in multistable delay-differential equations and their singular limit maps, Physical Review E, 58, 410-422.
Just W., 1999, Principles of Time Delayed Feedback Control, In: Schuster H.G., Handbook of Chaos Control, WileyVch, ISBN 3-527-29436-8.

Kwon O.-J., 1999, Targeting and stabilizing chaotic trajectories in the standard map, Physics Letters A, 258, 229-236.

Ott E., C. Greboki, J.A. Yorke, 1990, Controlling Chaos, Phys. Rev. Lett. 64, 1196-1199.

Pyragas K., 1992, Continuous control of chaos by selfcontrolling feedback, Physics Letters A, 170, 421-428.

Pyragas K., 1995, Control of chaos via extended delay feedback, Physics Letters A, 206, 323-330.

Richter H. and K. J. Reinschke, 2000, Optimization of local control of chaos by an evolutionary algorithm, Physica $D, 144,309-334$.

Zelinka I., 2004, SOMA - Self Organizing Migrating Algorithm, In: New Optimization Techniques in Engineering, (B.V. Babu, G. Onwubolu (eds)), chapter 7, 33, Springer-Verlag, ISBN 3-540-20167X

Zelinka I., 2005a, Investigation on Evolutionary Deterministic Chaos Control - Extended Study, In: ECMS 2005, Riga, Latvia, in June 1-4, 2005

Zelinka I., 2005b, Investigation on Evolutionary Deterministic Chaos Control, IFAC, Prague 2005

\section{AUTHORS BIOGRAPHIES}

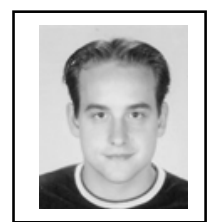

ROMAN SENKERIK was born in the Czech Republic, and went to the Tomas Bata University in Zlin, where he studied Technical Cybernetics and obtained his degree in 2004. He is now a Ph.D. student (Control of Chaos by Evolutionary Algorithms) at the same university.

Email address: senkerik@fai.utb.cz

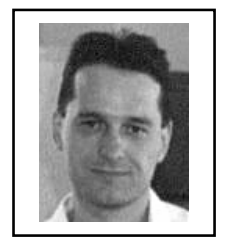

IVAN ZELINKA was born in the Czech Republic, and went to the Technical University of Brno, where he studied Technical Cybernetics and obtained his degree in 1995. He obtained Ph.D. degree in Technical Cybernetics in 2001 at Tomas Bata University in Zlin. Now he is an associated professor (Artificial Intelligence, Theory of Information) and head of department.

Email address: zelinka@fai.utb.cz Web-page can be found at: http://www.ft.utb.cz/people/zelinka.

EDUARD NAVRATIL was born in the Czech Republic, reached his master degree in Mathematical Engineering at the Brno University of Technology in 2003. He is currently a Ph.D. student (Information Flow in Complex Systems and Their Control) at Tomas Bata University in Zlin.

E-mail address: enavratil@fai.utb.cz 